\title{
IR thermography system for control and monitoring of energy saving and safety in heating and electrical equipment of housing services
}

By I. Petrosyants

Dept. of Electronics and Electrical Engineering, Moscow State Institute of Electronics and Mathematics (Technical University), B. Trekhsvyatitelsky Lane 3, 109028 Moscow, Russia, eande@miem.edu.ru

\begin{abstract}
IR thermography system is applied to housing services for control and monitoring of:

1) heat losses in buildings through windows, doors, floor and ceiling joints, heating systems;

2) energy saving and safety of electrical equipment: motors, transformers, engines, wiring and others;

3) hidden objects: wiring and underfloor heating systems. developed.

Technique for processing and software tool for visualization of the results of thermal measurements are
\end{abstract}

\section{Introduction}

Energy saving and safety in housing service systems are the global human problems. A portion of energy spent on heating of buildings is considerably lost. These losses can be the result of: heat losses through windows and glazed areas, doors; low-quality thermal insulation of building structures; defects in the masonry; disturbance of heating systems (including radiators, piping systems, floor heating, etc.).

Other objects of energy losses are electric equipment, wiring and electronic devices. Overheating increases power and energy losses, reduces reliability and service life of equipment, sometimes being the cause of emergency situations. The diagnosis of temperature of electric and electronic systems is really important and urgent task especially for hidden objects like wiring, underfloor heating systems, etc.

Several Infrared control and monitoring systems are used for building and renewable energy applications [1, 2], inspecting electric motors [3] and other objects [4]. But they do not take into account all aspects and details of housing services objects and electrical equipment inspection. presented.

In this paper the IR thermography system oriented for control and monitoring of housing services objects is

\section{Structure of Infrared control and monitoring system}

The developed Infrared control and monitoring system consists of three main blocks (see Fig. 1).

Hardware block is based on A40 FLIR Systems Infrared camera that is connected to laptop by FireWire interface. In «Hardware»-block are also used: portable printer (for rapid delivery of results); camera (for taking photos of objects and areas are being investigated and their subsequent comparison with the obtained thermograms); tripod (for infrared camera's motionless fixation).

Software and regulations block of the system is based on the standard and self-made software tools.

Standard ThermaCAM Researcher software is used for IR camera control, visualization, processing and analysis of obtained data.

Self-made techniques and developments are significant parts of the developed system and included:

- $\quad$ special technique for processing and software tool for visualization of the results of thermal measurements;

- techniques for hidden objects (wiring and underfloor heating systems) investigation.

Measured object description block is responsible for the correct choice of objects to be investigated. Depend on object's type different software and regulations should be used.

Thus, developed Infrared control and monitoring system is used for solving three main problems:

1. Identification and localization of heat losses in buildings through windows and glazed areas, doors, floor and ceiling joints.

2. Analysis of electrical and electronic equipment thermal regimes and exposure of local overheating problems («hot spots»).

3. Safety control of electric wiring and components to prevent the fire and hazardous situations. 


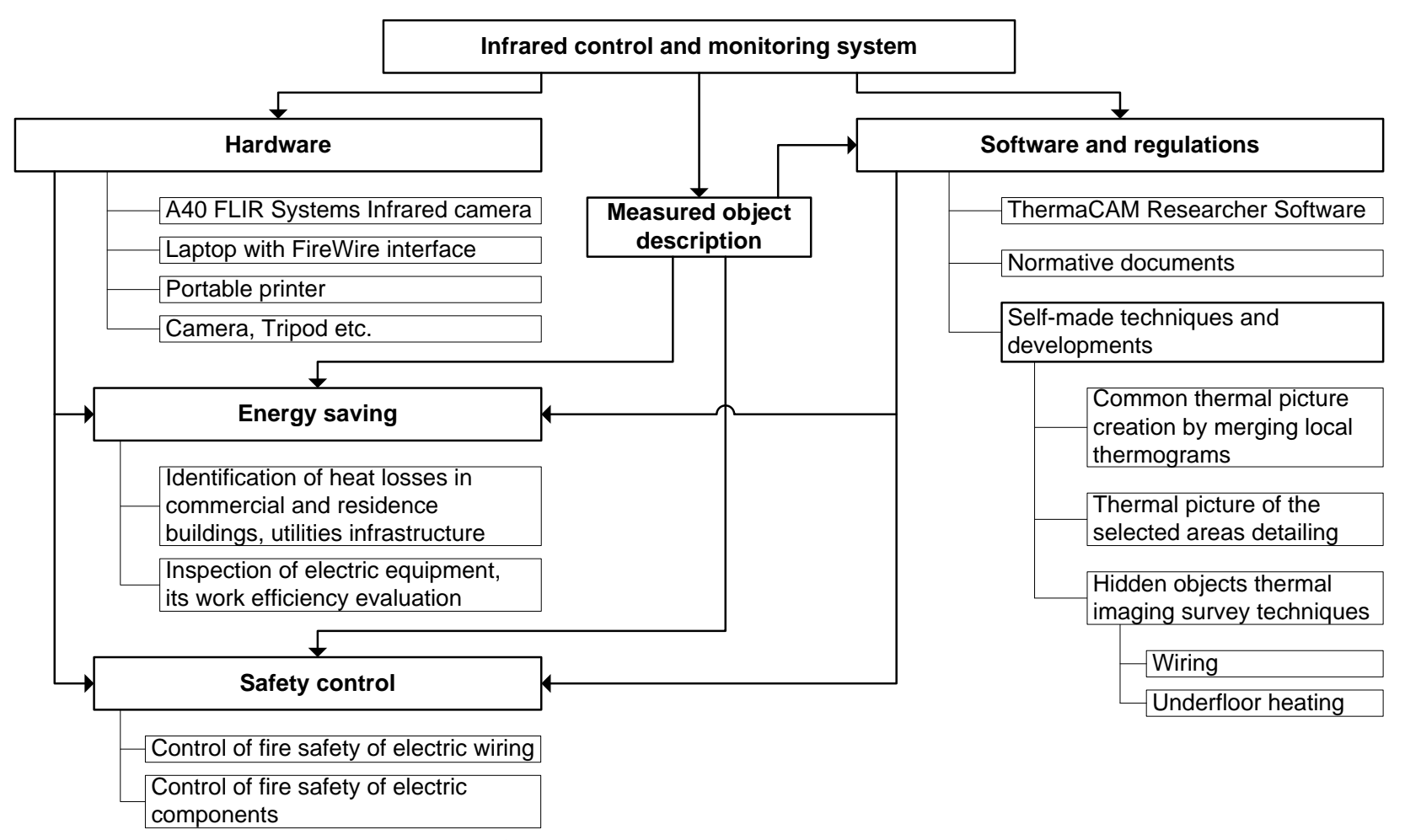

Fig. 1. Structure of Infrared control and monitoring system.

\section{Applications}

Different examples of heating and electrical equipment investigation are presented. Based on analysis of obtained thermograms the recommendations for defects elimination and energy losses minimization are given.

\subsection{Temperature inspecting of buildings}

There is a great deal of objects in buildings that can be interesting for thermal measurements: glazed areas, doors, floor and ceiling joints, heaters and heating systems, etc. It can be very useful to link the results of thermal measurements of individual objects to their location on the drawings and plans.

For solving described problem special software tool «ImgPresenter» is developed. «ImgPresenter» is used for visualization of thermal measurements results with its linking to investigated objects, areas or drawings and plans.

Main features of «lmgPresenter»:

- Creation a new project and opening an already created project;

- Saving project in format of the developed program *.ipsr;

- Background image selection (e.g. investigated object or plan of the premises);

- Selection, editing and deleting necessary areas on the background image;

- Linking to areas the results of thermal measurements (thermograms, graphic representations, Microsoft Word document, Microsoft Excel document, text file, etc.);

- Customization of the selected areas: the choice of color and line thickness of selected areas;

- Adding comments to selected on the background image areas that appear when directing the mouse cursor over the area.

Two versions of «ImgPresenter» software tool are developed. «Editor's version» provides all the features described above. The second one «ImgPresenter user's version» allows only to work with the project which has already been created using «Editor's version» (without possibilities to make new or edit any projects), this special version is passed to the customer or builders.

Fig. 2 illustrates the example of «lmgPresenter» usage. After thermal measurements in the building are performed its results can be linked to floor plan of the building and visualized using «ImgPresenter» software tool. All investigated objects (windows, doors, heaters, electrical units, etc.) are marked on their place on the floor plan and linked with corresponding to them result (e.g. thermograms, diagrams, some notes, etc.).

Legend for Fig. 2:

1. Marked on the floor plan of the building areas, where in the course of thermal imaging survey defects were revealed;

2. A floor plan of the building that is loaded into the program «ImgPresenter» as a background image; 
3. Photo of area being investigated with corresponding to it thermogram that appeared by selecting area;

4. Chosen on the floor plan area;

5. Comment that appears when directing the mouse cursor over the area.

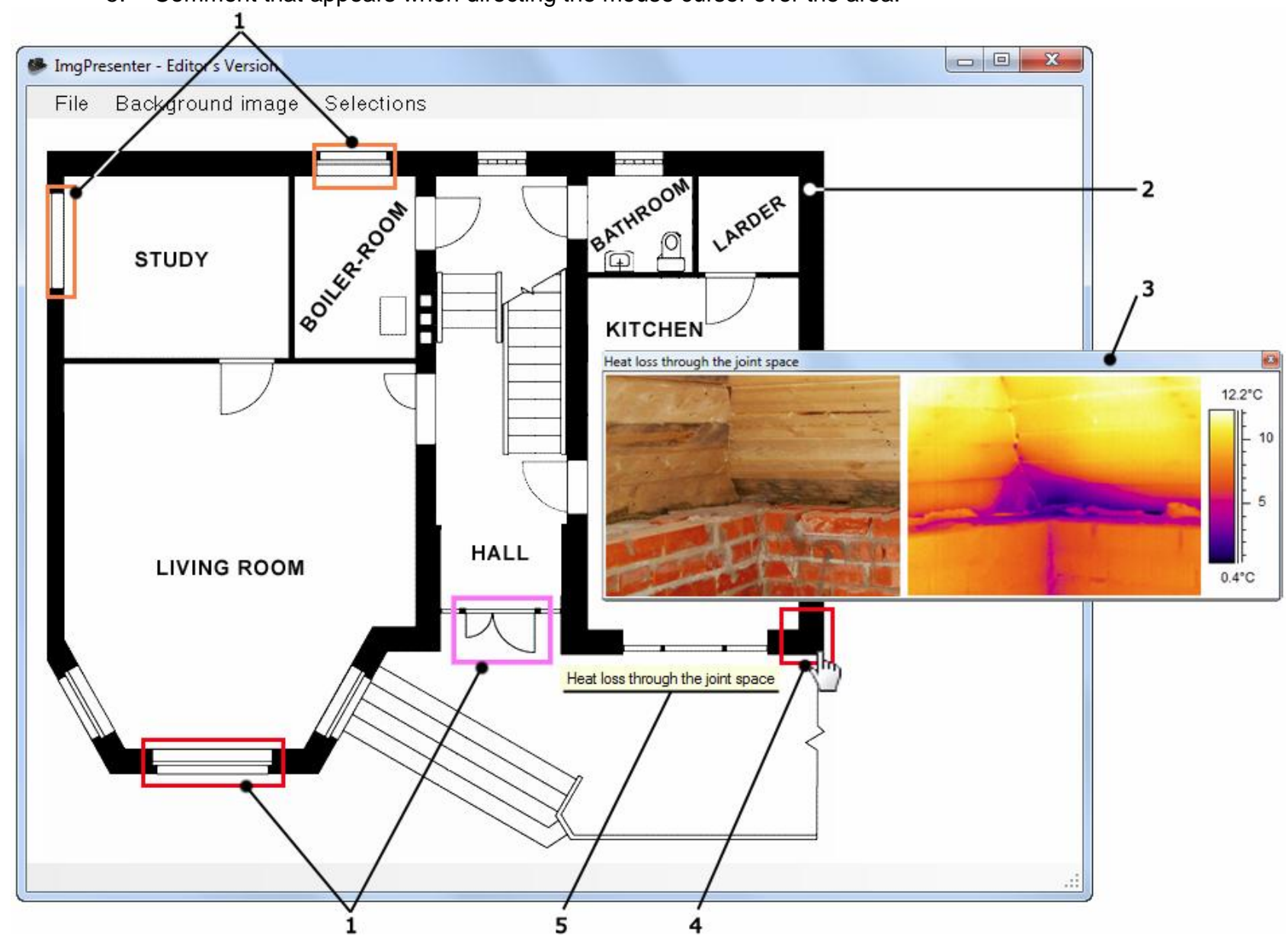

Fig. 2. Main window of «ImgPresenter» software tool.

\subsection{Heat losses through windows}

Sometimes objects to be investigated are not completely in FOV (field of view) of Infrared camera. It can be useful to have common thermal pictures of such objects for better understanding the results of thermal measurements. However, not all Infrared cameras and their software are available to create common thermal picture of large area objects. So it is necessary to merge a set of obtained local thermograms.

Investigation of windows is the quite typical example of such situation that can take place in the buildings.

So a few modern software products which can merge pictures were analyzed and the algorithm of choosing software which is the best to a local situation (depending on different criteria) is developed (Fig. 3).

These software products are:

- Adobe Photoshop CS3 (raster graphics editor);

- PTgui 8.2.1. Pro (software for merging photos);

- The Panorama Factory v.4.4 (software for merging photos);

- Microsoft ICE (freeware software for merging photos).

Criteria are being analyzed: dependence on the size of local thermograms to be merged, necessity to merge images both vertically and horizontally (mosaic mode), manual or automatic mode of merging, necessity of subsequent processing of the received images, efficiency of reception of final results.

Before thermograms are ready for merging it is necessary to preprocess them. So preprocessing method of local thermograms for merging is also developed. It is important to note that the temperature scales of local thermograms may be different because of the auto adjust scale function is enabled when thermal measurements are made. Thus, the entire area of the object being investigated should be surveyed to identify values of the maximum and minimum temperature, and then set these values as the upper and lower limits of the temperature scale of all local thermograms 
for the correct merging of several thermograms in the common thermal picture. Moreover, it is important to determine just the temperature of the investigated object, except the temperature of other objects which may also be in field of view of IR camera, which provides later more clearly and contrast common thermal picture of the object.

It was decided to use the video mode in radiometric format with a certain value FPS (Frames per second) to receive a set of thermal images suitable for subsequent pasting in various programs. In carrying out the actual work it was found that FPS $\geq 10$ is the most suitable. Next using ThermaCAM Researcher splitting of obtained video into scenes is made. Unlike usual photos, in the case of thermal images it is recommended that the percentage of overlapping frames should be quite high (sometimes up to $70-80 \%$ ), because such type images are less contrasty and have fewer binding control points.

So, it was found that for merging one row of thermograms (only in horizontal or vertical direction) in automatic mode The Panorama Factory is better than other programs being used. The main requirement for thermal images for merging is their size - it should be the same for all images. The Panorama Factory provides a range of options for merged images editing, but in failing to edit obtained image in this program it is recommended to make the subsequent processing in the software package Adobe Photoshop CS3.

For PTgui and Microsoft ICE the size of the images loaded for merging is not critical. In addition, these programs allow merging images in automatically "mosaic" mode. PTgui merges qualitative panoramas in automatic mode from usual photo images, but this software often can't find the control points for merging thermal images due to the nature of this type of image (specific color palette, low contrast), so it is necessary to do it by hand. Therefore, in many cases it may be preferable to use Microsoft ICE software for merging thermograms in the automatic mode both in the vertical and horizontal directions.

The software package Adobe Photoshop CS3 allows merging thermograms in manual mode, but it requires experience and skills in this software environment. In addition, Adobe Photoshop CS3 allows editing and processing common thermal images obtained in three other programs.

The developed algorithm is shown in Fig. 3.

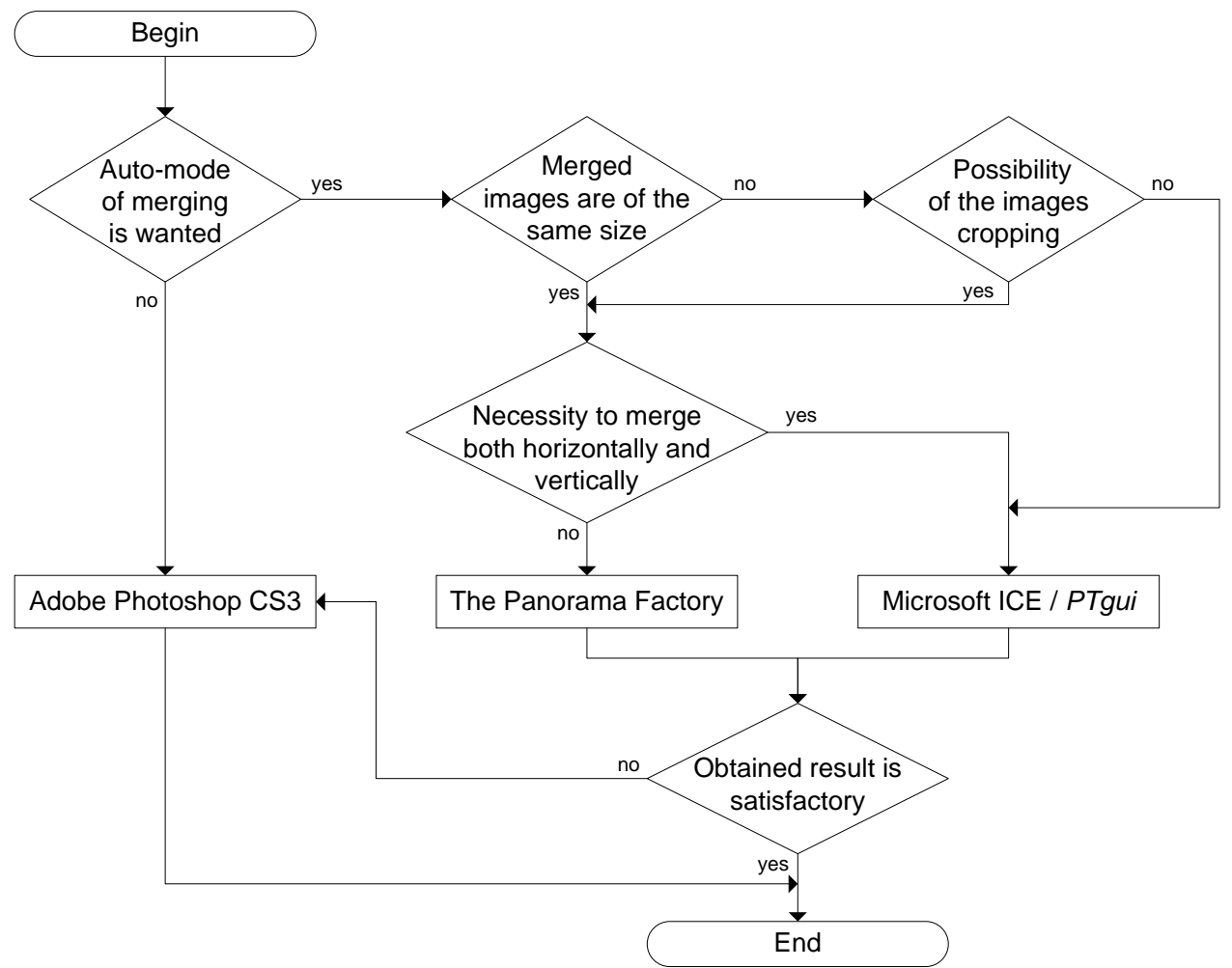

Fig. 3. Software selection algorithm for common thermal picture creation by merging local thermograms.

In Fig. 4(a) investigated window is shown. Also there are set of local obtained thermograms (Fig. 4(b)) and common thermal picture created with Adobe Photoshop CS3 usage (Fig. 4(c)). Note that each local thermogram from the shown set has its own temperature scale and it is necessary to adjust the same values of the temperature scale for all thermal pictures before merging can take place. 


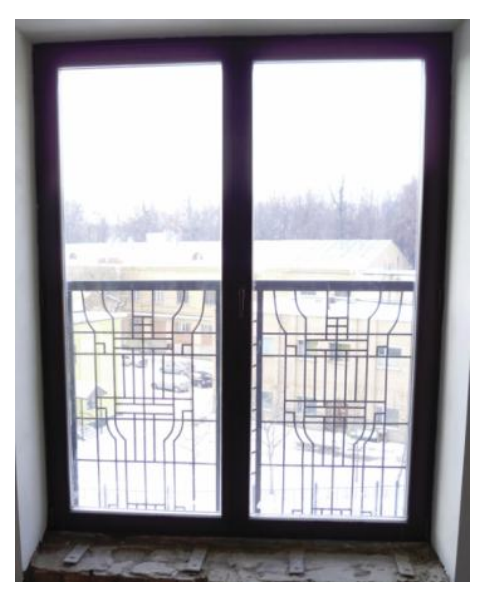

(a)

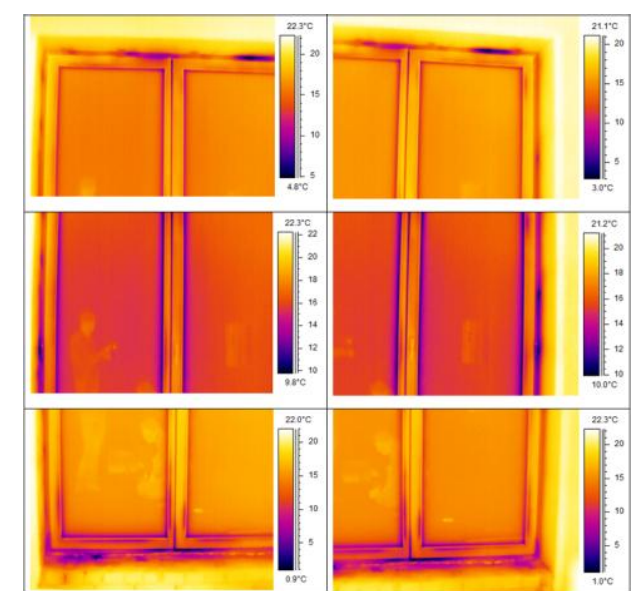

(b)

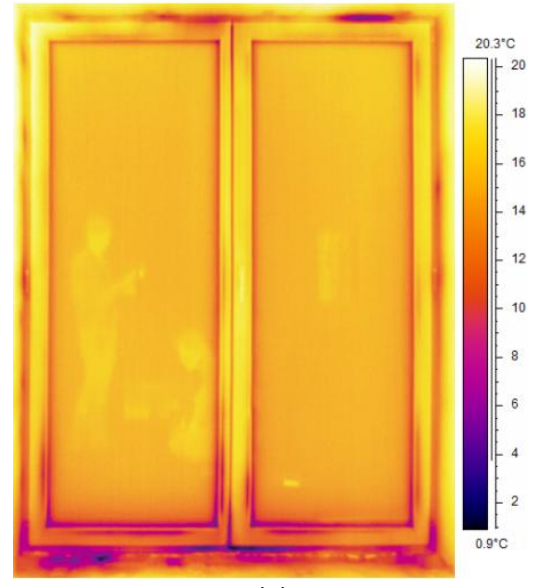

(c)

Fig. 4. Images of: investigated window (a), set of obtained local thermograms (b), created common thermal picture of the window (c).

So technique for obtaining and preprocessing the set of local thermograms of large-scale objects with its following merging for creating common thermal picture is developed. Algorithm for choosing software that is the best suitable for creating common thermal picture depending on different criteria is also developed. Thus, it possible to create more understandable thermal pictures of big squared objects.

\subsection{Motors and other electric equipment thermal regime control}

It is necessary to control motors, transformers, engines, etc. thermal regimes because overheating reduces reliability and service life of electric equipment and it also influences the power and energy losses. Moreover significant overheating can be the cause of emergency situations. According to equipment's passports conclusion about its work efficiency is given. The example of motor thermal measurement is shown in Fig. 5.
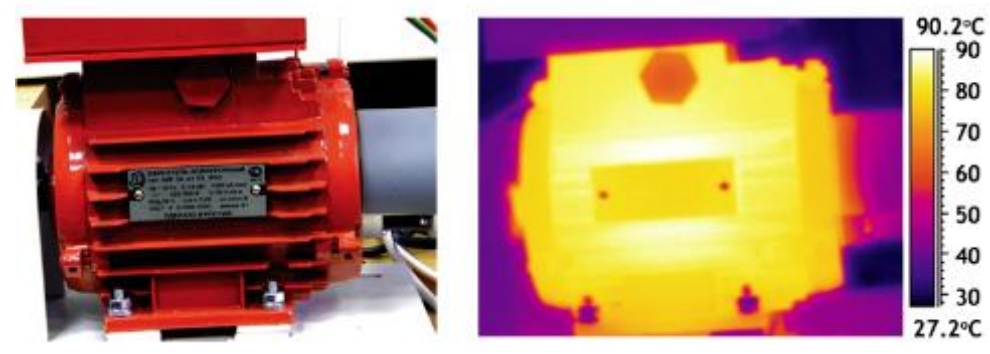

Fig. 5. Asynchronous motor AIR 56 A4, $P=0.12 \mathrm{~kW}, n=1500$ rotations $/ \mathrm{min}$, weight $3.18 \mathrm{~kg}$.

\subsection{Control of fire safety of electric components}

The diagnosis of temperature of electric components, wiring and elements of the electrical supply at the present time is really important and urgent task. Miscalculation of load on the system, installation defects, wear and tear and other factors can lead to overheating of electrical components and, therefore, be a source of emergency and fire situations.

When controlling electric components it can be the situation that there are few areas of one object which are interesting for analysis, but each area or one of them has a much different from other areas temperature scale. Thus, some areas on the obtained thermogram can be less contrast if the maximum temperature scale is selected. So thermal picture of the selected areas detailing can be very useful in this situation (illustrated in Fig. 6).

As it can be seen from the Fig. 6 (b) area which marked in Fig. 6 (a) as number 1 is less contrast against area number 2. It is the result of the big difference between values of these two areas temperature scales. In Fig 6 (c) and (d) thermal pictures of areas are shown. Each thermogram has its own temperature scale, so these thermal images are contrast and good for understanding.

Developed program «ImgPresenter» is used for solving such kind of problems too. It visualizes thermal measurements results with its linking to investigated object or area. 


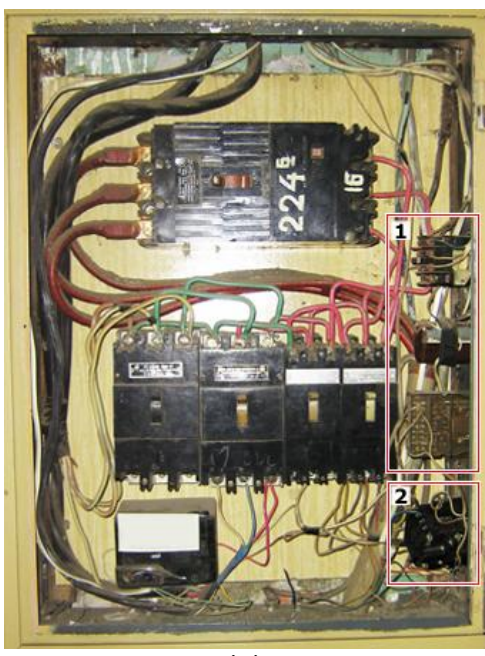

(a)

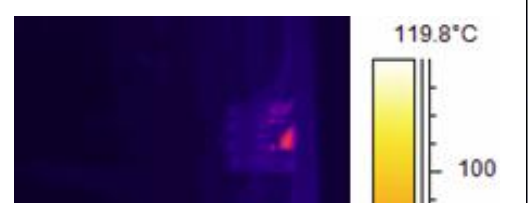

100

80

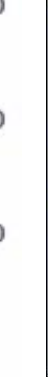

(b)

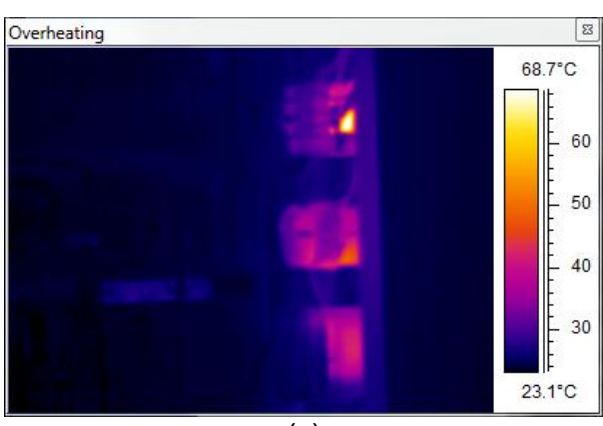

(c)

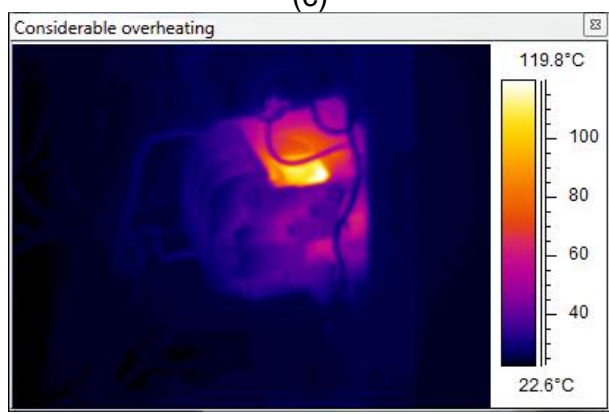

(d)

Fig. 6. Investigated switchboard: photo (a), obtained common thermal image of areas 1 and 2 (b), detailed visualization of areas 1 (c) and 2 (d).

\subsection{Hidden wiring control}

As far as wiring is hidden out of a sight and screened off (for example, by decorative paneling) developed technique is based on measuring temperature on an external surface of a wall: knowing material of covering and its thickness, distance from the heated wire, it is possible to restore the actual temperature of heating of hidden cables.

A special measuring stand (Fig. 7) was developed and used for research in this direction. It allows simultaneous measure temperature both of wiring located in the direct line of sight and on the surface of the decorative covering which hides wiring.

The laboratory transformer is powered from the mains. One output terminal of laboratory transformer is connected to $30 \mathrm{~A}$ ammeter (for measuring the input current) and then to the input terminal of the transformer; the other output terminal of laboratory transformer connects directly to the second input terminal of the transformer. From the transformer outputs one contact goes directly to the wire being investigated, the second - through 100A ammeter (for measuring the current supplied to the wire being investigated). The structure of the stand is shown in Fig. 8.

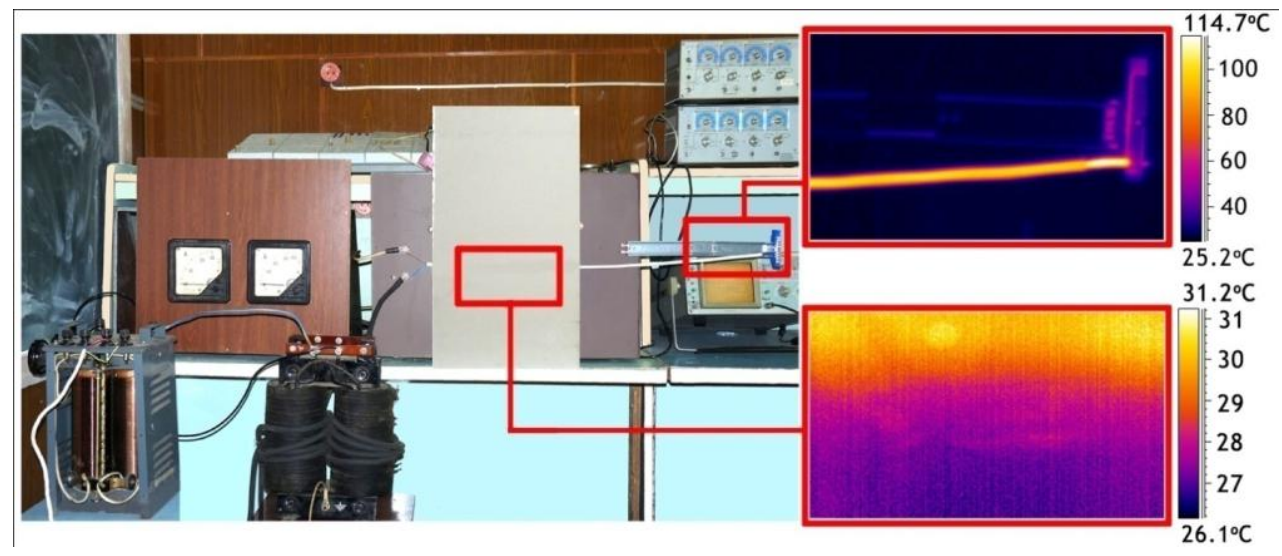

Fig. 7 The image of the stand for thermal regimes of hidden wiring research. 


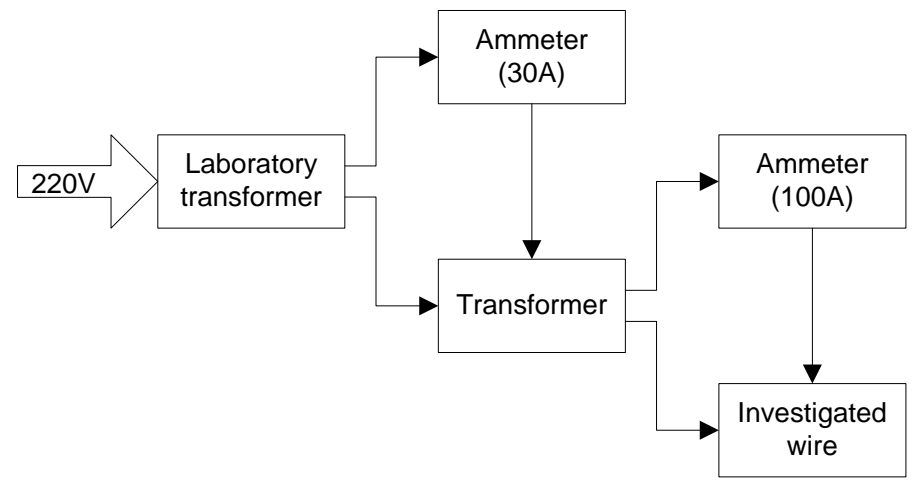

Fig. 8. The structure of the stand for thermal regimes of hidden wiring research.

Thus, the system is developed: laboratory transformer regulates the input voltage from 0 to $220 \mathrm{~V}$, the transformer reduces the voltage, increases current strength (magnification ratio can be adjusted by the number of secondary winding turns), as a result the circuit is closed on the transformer. Transformer is used in the stand can deliver currents up to $200 \mathrm{~A}$, that is sufficient for testing household wiring.

Research conducted for the most common cladding materials: drywall, PVC lining boards, ceiling and chipboard panels, for electrical wiring: PVS 2x1.5, PVPP $2 \times 1.5$ and PVSn $2 \times 2.5$. Tables of threshold values of temperature difference $\Delta T$ on the surface of the covering materials are made. If the temperature exceeds these values, then it is possible to conclude that abnormal functioning of the electric wiring takes place.

Table 1 shows an example of a of threshold values of temperature difference $\Delta T$ on the surface of the different covering materials that hide the PVS $2 \times 1.5$ wire.

Table 1. Threshold values of temperature difference $\Delta T$ on the surface of the different covering materials.

\begin{tabular}{|l|c|c|c|c|}
\hline Type of wire & \multicolumn{3}{|c|}{ PVS 2x1.5 } & Chipboard panels \\
\hline Material & Drywall & PVC lining boards & Ceiling panel & 1,5 \\
\hline $\begin{array}{l}\text { Threshold values of } \\
\text { temperature difference } \Delta \mathbf{T},{ }^{\circ} \mathbf{C}\end{array}$ & 2,4 & 2,4 & 2,4 & \\
\hline
\end{tabular}

The examples of dependence of wire's outer insulation heating and temperature difference $\Delta T$ on the surface depending on the current flowing through the wire are shown on two diagrams below (Fig. 9).

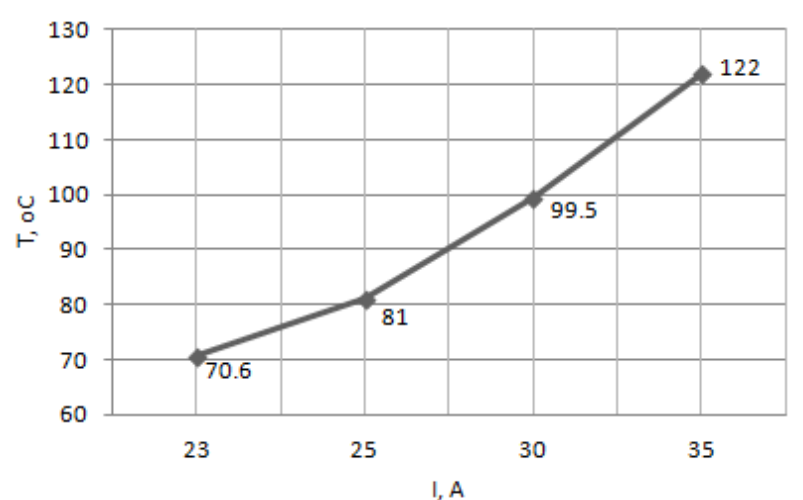

(a)

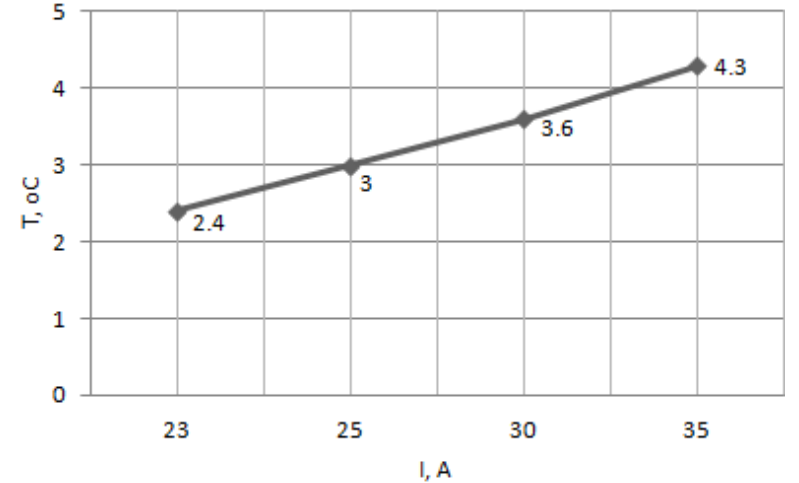

(b)

Fig. 9. Diagrams of: outer insulation heating of PVS $2 \times 1.5$ wire depending on the current flowing through it (a), temperature difference $\Delta T$ on the surface of drywall depending on the current flowing through PVS $2 \times 1.5$ (b).

Thus, the technique which allows controlling the state of hidden wiring is developed.

\subsection{Underfloor heating}

In modern buildings underfloor heating systems are often used. It is important to detect areas free of underfloor heating pipes before making various construction works. In this way the original technique of location search of underfloor heating pipes is developed. 
This technique includes following five steps:

1. Floor space is divided into areas where measurements will take place, these areas are marked on the plan of the premises;

2. Separation of areas being investigated using materials with low emissivity (e.g. aluminium materials) which will be clearly seen at obtained thermograms is made;

3. For each area photo and corresponding to it thermogram are saved - for more distinct interpretation of obtained thermogram directly at the placement where thermal measurement was made;

4. Thermograms merging (if required) for creation common thermal picture of large square area which is not completely in field of view of IR camera is made;

5. For each area regions free of underfloor heating pipes are determined;

Developed program «ImgPresenter» is used in this practical application too: the results of thermal measurements are linked to their location on the plan of the premises (Fig 10).

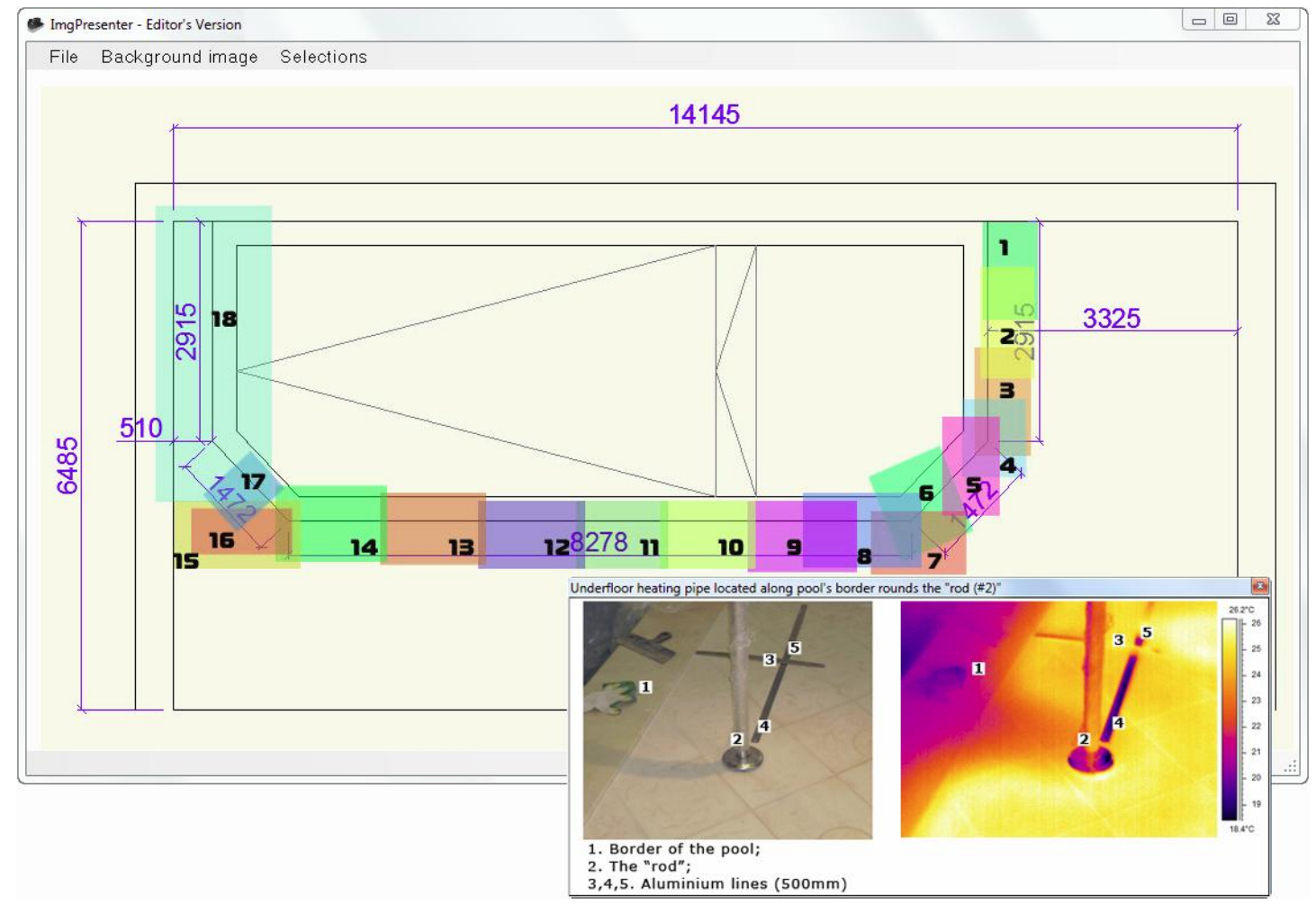

Fig. 10. Main window of «lmgPresenter» software tool with opened window of selected area visualization (on foreground).

As it can be seen from Fig. 10 the usage of «ImgPresenter» allows visualizing a great deal of areas right in one place all together.

Developed technique allows giving the results of thermal measurements to customers or builders in more understandable and easy-to-use form.

\section{Conclusion}

So Infrared thermography system for control and monitoring of energy saving and safety in heating and electrical equipment of housing services is developed.

Special software tool «ImgPresenter» which allows visualizing and detailing the results of thermal measurements with their linking to the investigated object or area or place on the plan of premises is developed. Thus, «ImgPresenter» allows storing the results of thermal measurements and making them available to the customer in more convenient, intuitive and informative form.

Technique of common thermal picture creation by merging local thermograms is developed. Several software products for merging pictures were analyzed into account of the thermal images specificity. Algorithm of choosing software which is the best to a local situation (depending on different criteria) is developed. developed.

Special techniques for identification and investigation of hidden wiring and underfloor heating systems are 


\section{Acknowledgment}

This work was supported by Russian Foundation for Basic Research, grants 10-07-00689 and 12-07-00506.

\section{REFERENCES}

[1] Vollmer M., Möllmann K.-P. "Infrared Thermal Imaging: Fundamentals, Research and Applications" Wiley-VCH, 1 edition, 2010.

[2] FLIR Systems AB "Thermal imaging guidebook for building and renewable energy applications", 2011.

[3] Fluke Corporation "Thermal Applications: Inspecting electric motors", 2008.

[4] Created in Cooperation with Fluke Corporation and The Snell Group "Introduction to Thermography Principles", 2009. 